\title{
The Role of Guanxi and Positive Emotions in Predicting Users' Likelihood to Click the Like Button on WeChat
}

\author{
Haichuan Zhao ${ }^{1 * t}$ and Mingyue Zhang ${ }^{2+}$ \\ ${ }^{1}$ School of Management, Shandong University, Jinan, China, ${ }^{2}$ Asia Europe Business School, Faculty of Economics \\ and Management, East China Normal University, Shanghai, China
}

\section{OPEN ACCESS}

Edited by:

Kostas Karpouzis,

Institute of Communication and Computer Systems, Greece

Reviewed by:

Barbara -

Lewandowska-Tomaszczyk State University of Applied Sciences

in Konin, Poland

James Alan Kole,

University of Northern Colorado,

United States

*Correspondence:

Haichuan Zhao

zhhc@sdu.edu.cn

tThese authors have contributed equally to this work and share first authorship

Specialty section: This article was submitted to Human-Media Interaction, a section of the journal Frontiers in Psychology

Received: 11 February 2020 Accepted: 23 June 2020

Published: 22 July 2020

Citation:

Zhao H and Zhang M (2020) The Role of Guanxi and Positive Emotions in Predicting Users' Likelihood to Click

the Like Button on WeChat.

Front. Psychol. 11:1736.

doi: 10.3389/fpsyg.2020.01736
Clicking the like button is a popularly used interaction function on social media. Although prior empirical studies have examined motives for liking behavior on social media, they have largely neglected culture-driven theories and the underlying mechanisms for why users click the like button. By integrating guanxi theory and the affective response model, we propose a conceptual model to determine what factors influence users' liking behavior on WeChat. We examine data from an online survey of 327 respondents using PLS-SEM. The findings show that content cues (i.e., content usefulness and content interestingness) positively affect WeChat users' positive emotions, which in turn predict their intention to like. More importantly, guanxi cues (i.e., mianzi giving, renqing, and ganqing) positively affect WeChat users' expected guanxi benefit, which in turn affects intention to like in two ways. On the one hand, the expected guanxi benefit has a direct positive effect on intention to like; on the other hand, this benefit can translate into positive emotions, which in turn predict users' intention to like.

Keywords: intention to like, guanxi, affective cues, positive emotion, expected guanxi benefit

\section{INTRODUCTION}

The advancement of the Internet has increased the popularity of social media. In turn, social media has significantly changed interpersonal interactions. The like button is a popularly used interaction function on social media (Basalingappa et al., 2016), such as Facebook, Twitter, Sina micro-blog, and WeChat, and other online platforms also provide this function. The like button was initially supposed to show users' positive emotions about the usefulness and interestingness of the content (Sun et al., 2016); however, with the increased usage of this button, the original meaning of "like" has changed. Many users now assume that the number of likes received from others is a reflection of reputation and social status. Thus, they also use the like button to maintain and strengthen relationships with others by showing support (Wang et al., 2016). By exploring the complicated motivations behind the use of the like button, this research tries to uncover the factors and mechanisms that influence people's use of this button on social media from a guanxi and emotional perspective.

Users' liking behavior on social media has received some attention in the literature under two main research streams. The first pertains to the consequences of users' liking behavior from a company marketing perspective. Prior research indicates that customers' use of the like 
button can increase their word-of-mouth and purchase intentions (Bunker, 2013; Mariani and Mohammed, 2014). The second stream involves users' motivations for clicking the like button from an interpersonal interaction perspective (Lee et al., 2016; Sun et al., 2016; Gan, 2017). For example, Sun et al. (2016) find that social value, entertainment value, and cognitive value are key determinants of WeChat users' liking behavior. Gan (2017) argues that user-perceived hedonic gratification, social gratification, and utilitarian gratification positively affect WeChat users' liking behavior. WeChat is the most popular form of social media in China. It provides multiple mobile services, including mobile communication services via text and voice and mobile social networking services. Although prior research has examined users' liking behavior in a Chinese context, it has neglected some key elements that may affect Chinese users' behavior, such as guanxi. The Chinese use WeChat not only to express their emotions when reading useful and interesting content but also to strengthen guanxi with their friends (Wang et al., 2016). Guanxi, or the existence of direct particularist ties between two or more individuals (Jacobs, 1979; King, 1991; Tsui and Farh, 1997), plays a major role in Chinese society (Alston, 1989) and is considered an important strategic asset by most Chinese people.

Although a few previous studies examined the impact of such social dimensions as social gratification and social value on WeChat users' liking behavior (Sun et al., 2016; Gan, 2017), guanxi is a conceptual construct that can be clearly distinguished from social gratification and social value. Social value refers to social benefits related to social interaction such as reciprocity, altruism, and expected relationship (Sun et al., 2016), whereas social gratification emphasizes maintaining social relationships by fulfilling social support by liking behavior in WeChat (Gan, 2017). In comparison, guanxi encompass three dimensions such as renqing (reciprocity), mianzi (face) giving, and ganqing (affect). Mianzi (face) giving refers to social image maintenance and construction by liking each other's post in WeChat and ganqing (affect) indicate that people use liking behaviors in WeChat to confirm the closeness of their relationships. Thus, guanxi goes beyond the conceptual scope of social value and social gratification, and furthered the field of liking behavior in WeChat by approaching it in the perspective of social image and affective concerns. Thus, we examine how guanxi affects users' liking behavior on social media. In addition, prior research only specifies the factors that affect users' likelihood to click the like button, while neglecting the underlying mechanisms that may affect users' liking behavior. This study aims to fill these gaps.

When people decide to click the like button, they are considering more than just how the content makes them feel. Their decision is also likely based on both cognitive factors (e.g., content quality, expected guanxi benefit) and affective factors (e.g., how it makes them feel, or positive emotions). Thus, building on the affective response model (ARM) and guanxi theory, this paper proposes an integrated conceptual model that shows how guanxi cues (i.e., mianzi giving, renqing, and ganqing), content cues (content usefulness and content interestingness), and expected guanxi benefit motivate users to click the like button due to positive emotions. We empirically test the model and hypotheses using data from a WeChat sample.

\section{THEORETICAL BACKGROUND AND RESEARCH HYPOTHESES}

\section{Linguistic and Individual's Behavior}

WeChat users often post information on WeChat Moments. Language, in a broad sense, is the way information is expressed. Users' language used in a post may express their idea, mood, and experience, and it may affect their friends' reactions to the post, such as clicking the "like" button, writing reviews, and re-post. Previous research indicated that the language will influence reader's social cognition and emotion, and further affect their behavior (Burke, 1966; Semin and Fiedler, 1988; Leiner et al., 1991; Pennebaker and Francis, 1996; Mascolo and Fischer, 2015). Furthermore, prior research suggested that people's cognition on language was also affected by their culture which was developed by historical circumstances (Gobel et al., 2011; Tajima and Duffield, 2012). In Chinese society guanxi is considered an important factor that shape Chinese's interaction with others, and their cognition, emotion, and behavior can be affected by guanxi. Thus, in our paper we chose guanxi cues (culture related cues) and content cues (linguistic related cues) as two kinds of antecedents of the behavior of clicking the "like" button.

\section{Affective Response Model (ARM)}

Affect is a critical factor in human decisions and behaviors in many social contexts. Zhang (2013) developed ARM to explain how people's affect influences their decisions and behavior within the information and communication technology (ICT) context. WeChat, a popular ICT in China, provides instant messages via text and voice and mobile social networking services (Che and Cao, 2014; Zhang et al., 2016; Gan, 2017; Gan and Li, 2018). Thus, we adopt ARM to explain users' liking behaviors in this context. ARM proposes that ICT stimuli (e.g., affective cues) can influence people's affective reactions (e.g., emotions). These affective reactions generate and arouse people's behavior in the ICT. Research also indicates that people make final decisions and correspondingly choose their behavioral responses under different emotional states, such as approach versus avoidance (Mehrabian and Russell, 1974). In the context of liking behavior, the reason people click on the like button is to show their appreciation of others or others' posts, and this positive emotion induces their liking behavior.

In the ARM, affective cues are "specific features or characteristics of a stimulus that can manifest the affective quality of the stimulus" (Valdez and Mehrabian, 1994; Zhang, 2013). Affective cues have been assessed as environmental cues or signals containing affective information that can influence emotions (Valdez and Mehrabian, 1994) and cognitive processing strategies (Soldat et al., 1997). The literature specifies that information characteristics (Wang et al., 2017), environmental characteristics (Valdez and Mehrabian, 1994), product characteristics (Graa and Danielkebir, 2012), and social relationship characteristics 
(Wang et al., 2017) are all distinctive affective cues. As mentioned previously, people use the like button not only to express their positive emotions about the usefulness and interestingness of the content but also to maintain and strengthen guanxi with others (Wang et al., 2016; Sun et al., 2016). Building on the literature on affective cues, we identify the affective cues that fit the context of WeChat and categorize them into two types: guanxi cues (i.e., mianzi giving, renqing, and ganqing) and content cues (i.e., content usefulness and content interestingness). We then explain our main reasons for choosing these characteristics.

\section{Guanxi Theory}

According to previous research, guanxi is a social exchange mechanism built on mutual favors (Lee et al., 2017). The concept of guanxi is rooted in Confucianism; it is considered an important strategic asset by most Chinese people and companies (Park and Luo, 2010; Lee et al., 2017). Guanxi has been examined in both business and interpersonal relationships. Previous studies in businesses have mainly identified guanxi networks as a resource for organizations through which potential buyers and sellers are identified in China (Park and Luo, 2010; Yen et al., 2017). That research has mainly focused on the outcomes of guanxi, such as business performance, relationship-specific investment, and knowledge sharing (Zhuang and You-Min, 2007; Park and Luo, 2010; Luo et al., 2012; Yen et al., 2017). Previous research in interpersonal relationship has mainly explored how to build interpersonal guanxi (Ying, 2002; Warren et al., 2004; Chen et al., 2012). These studies indicate that Chinese people often participate in social activities to develop their guanxi networks with those who have the same friends, the same hometown, the same school, and the same neighborhood (Lee et al., 2001). In the process of developing guanxi, the Chinese tend to engage in the appropriate actions to enhance and maintain their guanxi networks, such as mianzi (face) giving, renqing (favor) giving, and ganqing (affect) supporting (Hwang, 1997); in return, they also expect to receive the guanxi benefit in return when they engage in actions to develop guanxi. WeChat is a social platform on which the Chinese can maintain and enhance guanxi with others in their social networks (Wang et al., 2016). Thus, we identify users' guanxi motivation as an affective cue of positive emotions and intention to like.

\section{Guanxi Cues}

\section{Mianzi Giving and Expected Positive Guanxi Benefit}

Mianzi (face) refers to an individual's claimed sense of positive self-image of his or her social status (Lee and Dawes, 2005). In general, the Chinese are concerned about saving face themselves and also giving mianzi to others (Redding and Michael, 1983). Previous research indicates that mianzi can be shared (Haugh and Hinze, 2003). The Chinese often expect members of their social networks to give mianzi to one another (Cardon and Scott, 2003). That is, it is the responsibility of all members in the network to enhance one another's status. Enhancing and maintaining mianzi, in turn, improves the guanxi between members (Cardon and Scott, 2003). On WeChat, many people consider that they have a great deal of mianzi when their posts receive many likes, and vice versa. The Chinese want to maintain or enhance the mianzi of others because providing no mianzi can destabilize or end the guanxi between members (Wang et al., 2016). Thus:

H1: Mianzi giving positively influences WeChat users' expected guanxi benefit.

\section{Renqing and Expected Positive Guanxi Benefit}

Renqing, or reciprocity, refers to one's obligation to return favors and show empathy to his or her personal relationship partner (Wang, 2007). More specifically, it means that if a personal relationship partner has received a favor, he or she must return the favor as soon as the opportunity arises (Hwang, 1987; Lovett et al., 1999). When both parties of a personal relationship engage frequently in the exchange of renqing, their guanxi will be strengthened. On WeChat, users can give their social network partner a favor by clicking the like button. They may also be recipients of others' likes by engaging in liking behavior for others. The rules of reciprocity in liking behavior thus enhance users' guanxi with others.

$\mathrm{H} 2$ : Renqing positively influences WeChat users' expected guanxi benefit.

\section{Ganqing and Expected Positive Guanxi Benefit}

Ganqing (affect), which is an indicator of the closeness of guanxi, refers to the feelings of emotional commitment among members of a social network (Mei-hui Yang, 1994). It is a form of social capital that provides leverage in interpersonal exchanges of favors (Yen et al., 2011). Ganqing can be built by sharing feelings with others about common topics, such as hobbies, experiences, beliefs, judgments, and opinions (Chen et al., 2004). On WeChat, if others share their experiences, opinions, and so on, in their posts, clicking the like button means that users endorse the content in those posts; thus, two parties can obtain ganqing on a long-term basis through liking behavior.

H3: Ganqing positively influences WeChat users' expected guanxi benefit.

\section{Content Cues}

Many users use WeChat to obtain useful and helpful content (Gan and Li, 2018). Previous research indicates that the content of a post is the key determinant of people's choice of behavior toward that post, such as reading, writing a review, reposting, and clicking the like button (Zhao et al., 2016a,b). Gan (2017) shows that useful content seeking motivates users' liking behavior; thus, content is an important cue that influences users' liking behavior. Zhao et al. (2016a) proposes that users evaluate the content from two aspects: usefulness and interestingness. Accordingly, we chose these two aspects as affective cues that influence users' emotions and liking behavior.

Content usefulness refers to the degree to which an online post provides useful content that gives economic or functional benefits to users (Zhao et al., 2016a). That is, useful content satisfies users' needs. According to ARM, positive emotions typically arise as reactions to stimuli in an individual's environment that he or she appraises as satisfying his or her needs, goals, 
or concerns (Zhang, 2013). Following this logic, we propose that that usefulness of content information as an environmental stimulus induces positive emotions and thereby promotes liking behavior on WeChat.

Content interestingness is the extent to which the content provided by a poster is enjoyable in its own right apart from content usefulness (Olney et al., 1991; Zhao et al., 2016a). On WeChat, users read others' posts for pleasure and joy, and this enjoyable content can satisfy their entertainment needs. Previous research indicates that users' positive emotional states are aroused when they experience enjoyment (Olney et al., 1991). Thus, the entertainment benefits obtained from content cause users to experience positive emotional changes, which in turn promote their liking behavior.

H4: Information usefulness influences WeChat users' positive emotions.

H5: Information interestingness influences WeChat users' positive emotions.

\section{Positive Emotions and Like Intention}

Users' liking behavior on social media is often aroused by emotion (Sun et al., 2016; Gan, 2017). That is, an important reason for clicking the like button on social media is to express aroused emotional responses (Sun et al., 2016). According to ARM, affective cues can influence people's emotions and subsequently generate or arouse people's behavior tendencies (Zhang, 2013). In other words, emotional response mediates the impact of affective cues on behavior tendencies. Thus, users' decision making and behaviors will differ when their emotional states differ. In social media, people click Like button to show their appreciation to others or their posts, thus, positive emotion induce his/her Liking behavior. Positive emotions refer to "the extent to which a person feels enthusiastic, excited, and inspired" (Verhagen and Dolen, 2011). These emotional responses are widely considered key predictors of people's behavior. For example, Wang et al. (2017) find that positive emotions positively affect users' informationsharing behavior. Verhagen and Dolen (2011) show that positive emotions can improve customers' purchase intentions. In line with these studies and ARM, we propose the following:

H6: Positive emotions positively influence WeChat users' intention to like.

\section{Expected Guanxi Benefits From Positive Emotions and Like Intention}

In addition to expressing positive emotions, users click on the like button to enhance and maintain guanxi with others (Wang et al., 2016). Thus, expected guanxi benefit is also a key predictor of liking behavior. Expected guanxi benefit reflects the degree to which an individual can maintain or improve interpersonal guanxi through liking behavior (Bock et al., 2005). People often use social media to enhance and improve their relationship with others, and social media platforms provide many functions to help them do so, including the ability to click the like button, write reviews, and send instant messages via text and voice (Wang et al., 2016). As noted previously, many users treat the number of likes received from others as a reflection of their reputation and social status (Sun et al., 2016); thus, people often use the like button to show their support of others or others' posts, enabling them to maintain and enhance guanxi with others. We propose that users may engage in liking behavior when they expect to obtain a high guanxi benefit through such behavior.

Expected guanxi benefit also acts as an affective cue that may influence positive emotions. Previous research indicates that individuals will feel positive emotions about a behavior when they can benefit from that behavior. For example, Ajzen and Fishbein (1980) show that an individual's positive emotions become aroused when he or she gains benefits from social behavior.

Thus, we propose the following:

H7: Expected guanxi benefit positively influences WeChat users' liking intention.

H8: Expected guanxi benefit positively influences WeChat users' positive emotions.

Figure 1 shows the conceptual model of this study.

\section{METHODOLOGY}

\section{Measurement}

Our research model consists of eight multi-item constructs, and 28 measurement items were generated (see Table 1). We developed all instruments by adapting existing validated instruments when necessary. In particular, we adapted the measurement scales for the three dimensions of guanxi (mianzi giving, renqing, and ganqing) from Lee and Dawes (2005) and Nie et al. (2011). Scales to measure content usefulness came from Sussman and Siegal (2003), and those for content interestingness came from Olney et al. (1991). Items for expected guanxi benefit came from Hsu and Lin (2008). We adapted items of positive emotion from Beatty and Ferrell (1998). Finally, we adapted items of intention to like from Verhoef et al. (2002). All items were measured on a 7 -point Likert scale ( $1=$ strongly disagree, $7=$ strongly agree).

\section{Data Collection}

The online survey took place in October 2017 on WeChat. The survey was available on the website for a 2-month period and ended when no new responses were generated (see Appendix A). We recruited $10 \mathrm{WeChat}$ users to independently send invitation messages with a link to the online questionnaire to their friends on WeChat. At the same time, their friends also were asked to repost the survey on their social networks. Participants were directed to read a short description of study when they opened the link, then they were asked launch their WeChat and click into the Moments on Discover page. They were then asked to read the latest post in the Moments, go back to the survey and answer questions. To encourage participation, valid respondents were offered incentives of $10 \mathrm{RMB}$ to complete the survey. In total, 365 respondents completed the questionnaires. We checked all responses and dropped 23 responses that had the same score for all items. 


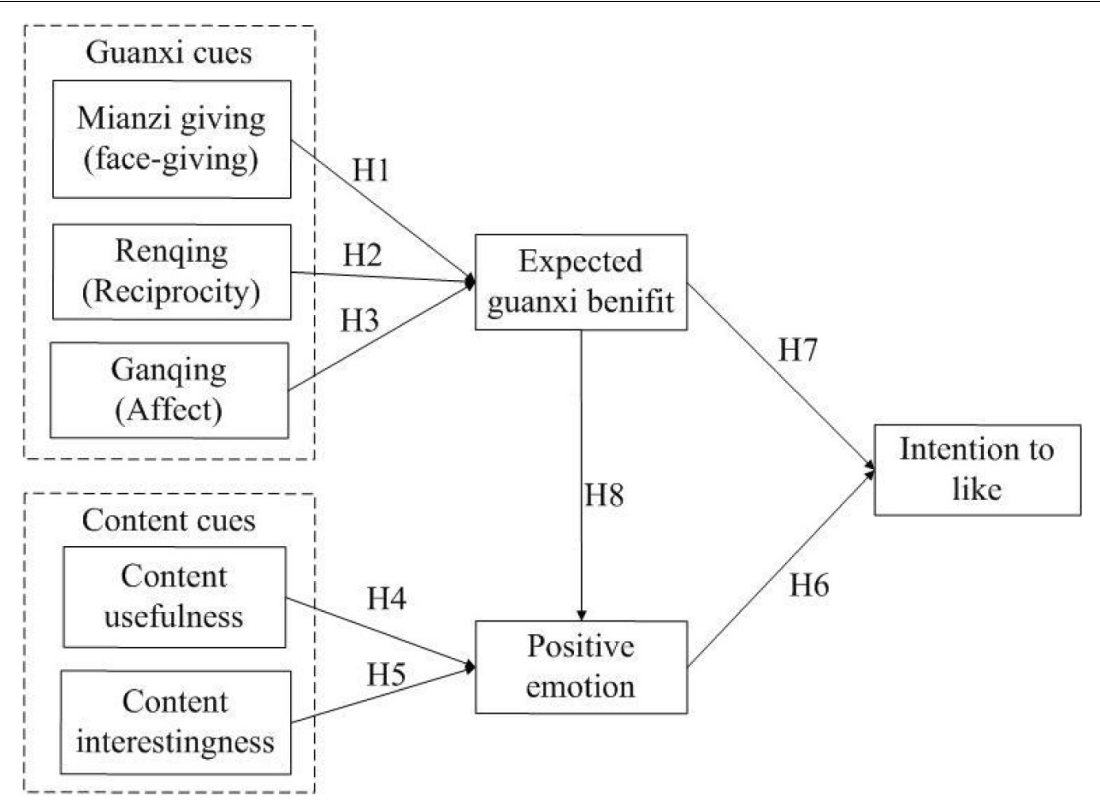

FIGURE 1 | Conceptual model.

TABLE 1 | Constructs and measures.

\begin{tabular}{|c|c|c|}
\hline Constructs & Measures & Sources \\
\hline & M2. The more "likes" we receive, the more Mianzi (face) we have. & Nie et al., 2011 \\
\hline \multirow[t]{2}{*}{ Renqing (R) } & $\begin{array}{l}\text { R1. In the WeChat, we will do this friend a favor by clicking like button if he/she did one for us } \\
\text { before. }\end{array}$ & $\begin{array}{l}\text { Lee and Dawes, 2005; } \\
\text { Nie et al., } 2011\end{array}$ \\
\hline & $\begin{array}{l}\text { R2. In the WeChat, this friend will do us a favor by clicking like button if we did one for him/her } \\
\text { before. }\end{array}$ & \\
\hline \multirow[t]{2}{*}{ Ganqing (G) } & $\begin{array}{l}\text { G1. This friend is my closeness friend, and we care for each other wholeheartedly } \\
\text { G2. I like this friend, and he/she likes me. }\end{array}$ & $\begin{array}{l}\text { Lee and Dawes, 2005; } \\
\text { Nie et al., } 2011\end{array}$ \\
\hline & $\begin{array}{l}\text { G3. I would try my best to help this friend when he/she is in need because he/she is a closeness } \\
\text { friend of mine. }\end{array}$ & \\
\hline Usefulness $(U)$ & $\begin{array}{l}\text { U1: The information in this post is important. } \\
\text { U2: The information in this post is informative. }\end{array}$ & $\begin{array}{l}\text { Sussman and Siegal, } \\
2003\end{array}$ \\
\hline \multirow{3}{*}{ Interestingness (I) } & 12: The information in this post is boring. & \\
\hline & I3: The information in this post is interesting. & \\
\hline & 14: The information in this spot is attractive. & \\
\hline \multirow[t]{2}{*}{$\begin{array}{l}\text { Expected guanxi } \\
\text { benefit (EGB) }\end{array}$} & $\begin{array}{l}\text { EBG1: Clicking like button on a friend's post in WeChat will strengthen the guanxi between us. } \\
\text { EBG2: Clicking like button on a friend's post in WeChat will create new guanxi with him/her. }\end{array}$ & Hsu and Lin, 2008 \\
\hline & EBG3: Clicking like button on a friend's post in WeChat will increase the trust between us. & \\
\hline \multirow{2}{*}{$\begin{array}{l}\text { Positive emotion } \\
\text { (PE) }\end{array}$} & PE1. When clicking like button on that post in WeChat, I was excited. & Beatty and Ferrell, 1998 \\
\hline & $\begin{array}{l}\text { PE2. When clicking like button on that post in WeChat, I was I was enthusiastic. } \\
\text { PE3. When clicking like button on that post in WeChat, I was inspired. }\end{array}$ & \\
\hline \multirow[t]{2}{*}{ Intention to like (IL) } & IL1. I am likely to click like button on this post. & Verhoef et al., 2002 \\
\hline & IL2. I would love to click like button on this friend's post continuously. & \\
\hline
\end{tabular}


TABLE 2 | Demographics of the research sample.

\begin{tabular}{|c|c|c|c|}
\hline Variable & & Count & $\%$ \\
\hline \multirow[t]{2}{*}{ Gender } & Male & 183 & 56.0 \\
\hline & Female & 144 & 44.0 \\
\hline \multirow[t]{5}{*}{ Age } & 17 or bellow & 6 & 1.8 \\
\hline & $18-30$ & 154 & 47.1 \\
\hline & $31-40$ & 103 & 31.5 \\
\hline & $41-50$ & 60 & 18.3 \\
\hline & $>51$ & 4 & 1.2 \\
\hline \multirow[t]{4}{*}{ Education level } & Junior high school & 10 & 3.1 \\
\hline & Senior high school & 15 & 4.6 \\
\hline & Undergraduate & 262 & 80.1 \\
\hline & Postgraduate & 40 & 12.2 \\
\hline \multirow[t]{5}{*}{ Monthly income } & $<2,000$ Yuan & 32 & 9.8 \\
\hline & 2,000-3,999 Yuan & 97 & 29.7 \\
\hline & 4,000-5,999 Yuan & 101 & 30.9 \\
\hline & 60,000-7,999 Yuan & 71 & 21.7 \\
\hline & $>\geq 8,000$ Yuan & 26 & 8.0 \\
\hline \multirow[t]{7}{*}{ Occupation } & Student & 65 & 19.9 \\
\hline & Company employee & 93 & 28.4 \\
\hline & Employee in public institutions & 84 & 25.7 \\
\hline & Civil servant & 33 & 10.1 \\
\hline & Self-employed & 38 & 11.6 \\
\hline & Retired & 10 & 3.1 \\
\hline & Others & 4 & 1.2 \\
\hline \multirow[t]{5}{*}{ Length of use } & $<6$ months & 8 & 2.4 \\
\hline & $>6$ months and $<1$ year & 35 & 10.7 \\
\hline & $>1$ year and $<2$ years & 124 & 37.9 \\
\hline & $>2$ years and $<3$ years & 89 & 27.2 \\
\hline & $>3$ years & 71 & 21.7 \\
\hline Average minutes using & $<10$ min & 86 & 26.3 \\
\hline \multirow[t]{3}{*}{ WeChat per day } & $>10 \mathrm{~min}$ and $<30 \mathrm{~min}$ & 101 & 30.9 \\
\hline & $>30 \mathrm{~min}$ and $<60 \mathrm{~min}$ & 104 & 31.8 \\
\hline & $>60 \min$ & 36 & 11.0 \\
\hline
\end{tabular}

We deleted another 15 responses because of missing data. As a result, 327 valid responses were processed for further analysis. Table 2 presents the demographic information of the respondents.

\section{DATA ANALYSIS AND RESULTS}

We employed partial least squares-structural equation modeling (PLS-SEM) using SmartPLS 2.0 M3 to estimate the parameters in the measurement and structural part of the model. PLSSEM is a useful approach because of the minimal demands on measurement scales, sample size, and residual distributions. An additional benefit of PLS is its ability to screen out factors that have a negligible effect on the dependent variables. PLS-SEM is thus appropriate for this study.

\section{Analysis of Measurement Models}

Table 3 shows the results of the measurement models in PLS-SEM analysis. All item loadings were greater than 0.7 , with a significant $t$-value, and the average variance extracted (AVE) for every construct was greater than 0.5, indicating convergent validity of all constructs. All Cronbach's alpha and composite reliability (CR) values exceed the general acceptable value 0.7 , suggesting good reliability. Finally, as Table 4 shows, the square root of the AVE for each construct is greater than its correlations with other variables, demonstrating adequate discriminant validity of all the constructs.

\section{Structural Model Test}

Figure 2 shows the results of model testing, including path coefficients, the corresponding levels of significance, and variance explained. An adequate proportion (36\%) of the variance in intention to like is explained by the proposed model. As expected, positive emotions $(\beta=0.50, t=8.15, p<0.001)$ and expected guanxi benefit $(\beta=0.14, t=2.18, p<0.05)$ positively affect intention to like. Thus, $\mathrm{H} 6$ and $\mathrm{H} 7$ were supported. For the antecedents of positive emotions, expected guanxi benefit $(\beta=0.36, t=5.61, p<0.001)$, content usefulness $(\beta=0.21, t=4.25, p<0.001)$, and content interestingness $(\beta=0.29, t=5.77, p<0.001)$ have significant effects on positive emotions; thus, $\mathrm{H} 8, \mathrm{H} 4$, and $\mathrm{H} 5$ were supported. Overall, a significant proportion (56\%) of the variance in positive emotions is explained by all affective cues (i.e., expected guanxi benefit, content usefulness, and content interestingness). In addition, mianzi giving ( $\beta=0.16, t=2.98$, $p<0.05)$, renqing $(\beta=0.27, t=4.56, p<0.001)$, and ganqing $(\beta=0.29, t=4.53, p<0.001)$ exert significant effects on expected guanxi benefit, in support of H1, H2, and $\mathrm{H} 3$, respectively. The explained variance of expected guanxi benefit is $38 \%$.

\section{DISCUSSION}

This study tests a conceptual model that proposes that content cues (i.e., content usefulness and content interestingness) affect WeChat users' positive emotions, which in turn predict their intention to like. More important, this study proposes that guanxi cues (i.e., mianzi giving, renqing, and ganqing) positively affect WeChat users' expected guanxi benefit, which in turn affects intention to like in two ways. On the one hand, expected guanxi benefit positively influences intention to like directly; on the other hand, expected guanxi benefit translates into positive emotions, which in turn predict users' intention to like. The results provide support for all the research hypotheses. Specifically, positive emotions and expected guanxi benefit are two crucial facilitators of intention to like on WeChat. The findings corroborate previous assumptions that users click the like button not only to express their positive emotions about the content but also to maintain or enhance relationships with others. In terms of the antecedents, expected guanxi benefit, content usefulness, and content interestingness exert positive effects on positive emotions. In addition, mianzi, renqing, and guanxi have positive effects on expected guanxi benefit. 
TABLE 3 | Scale properties.

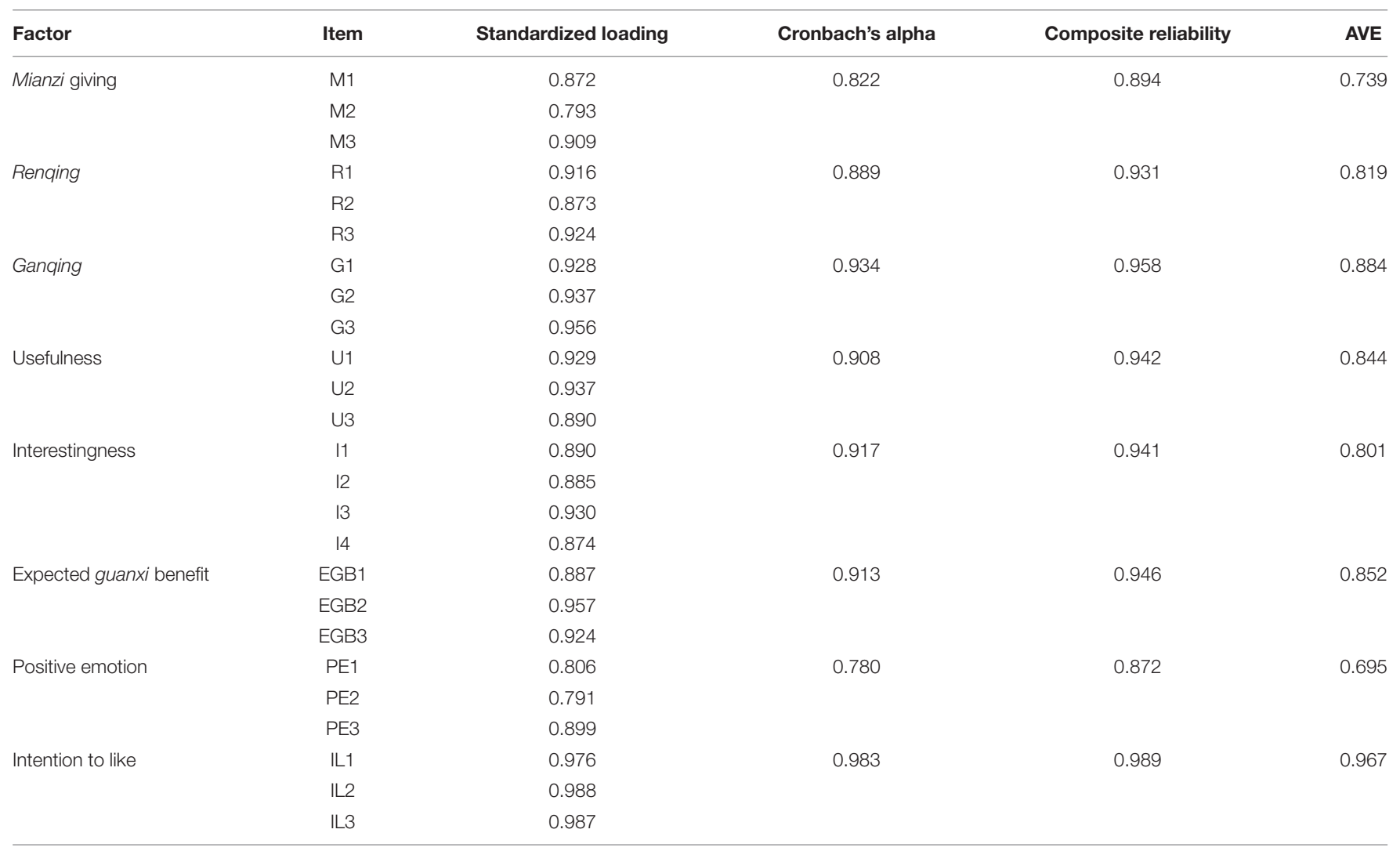

\section{Theoretical Implications}

This study contributes to extant literature in two ways. First, the Chinese use WeChat both to express their emotions when reading useful and interesting content and to strengthen guanxi with their friends. By contrast, prior research neglects the effect of guanxi on users' liking behavior (Mariani and Mohammed, 2014; Basalingappa et al., 2016; Sun et al., 2016; Gan, 2017). We show that guanxi cues (i.e., mianzi giving, renqing, and ganqing) motivate Chinese users to click the like button when they want to obtain a high level of the guanxi benefit through liking behavior. Thus, this study contributes to the literature in this field by treating guanxi as a key antecedent of liking behavior in the Chinese context.

TABLE 4 | Correlation coefficient matrix and square roots of AVEs.

\begin{tabular}{lcccccccc}
\hline & M & R & G & U & I & EGB & PE & IL \\
\hline M & 0.86 & & & & & & & \\
R & 0.51 & $\mathbf{0 . 9 0}$ & & & & & & \\
G & 0.56 & 0.66 & $\mathbf{0 . 9 4}$ & & & & & \\
U & 0.51 & 0.57 & 0.60 & $\mathbf{0 . 9 2}$ & & & & \\
I & 0.58 & 0.56 & 0.56 & 0.61 & $\mathbf{0 . 8 9}$ & & & \\
EGB & 0.46 & 0.54 & 0.56 & 0.63 & 0.64 & $\mathbf{0 . 9 2}$ & & \\
PE & 0.60 & 0.64 & 0.69 & 0.61 & 0.64 & 0.67 & $\mathbf{0 . 8 3}$ & \\
IL & 0.47 & 0.58 & 0.58 & 0.46 & 0.53 & 0.47 & 0.59 & $\mathbf{0 . 9 8}$ \\
\hline
\end{tabular}

Bolded values are the square roots of AVES.
Second, previous research mainly focuses on the factors that affect users' likelihood to click the like button, while overlooking the underlying mechanisms that may affect users' intention to click. This study aimed to fill these gaps by integrating guanxi theory and ARM to assess users' liking behavior. We proposed that positive emotions and expected guanxi benefit would arouse users' liking behavior. More specifically, we expected useful and interesting content to arouse users' positive emotions, which in turn motivate their intention to like. In addition, guanxi cues (i.e., mianzi giving, renqing, and ganqing) motivate Chinese users' liking behavior in two ways. On the one hand, they positively affect expected guanxi benefit and, in turn, influence intention to like directly. On the other hand, guanxi cues transfer to expected guanxi benefit and thereby produce positive emotions, in turn influencing intention to like. We enrich the literature on liking behavior by showing that positive emotions and expected guanxi benefit are the two underlying mechanisms of Chinese consumer liking behavior. In addition, we add to the literature by applying guanxi theory and ARM in a new context.

Third, previous research has focused on social benefits, social support and their impact on WeChat users' liking behavior (Sun et al., 2016; Gan, 2017). However, little research has approached liking behaviors on WeChat in a affective or social image perspective. The current research introduced the concept of guanxi, and examined mianzi (face) giving and ganqing (affect) on liking behavior in WeChat. The results indicated that people 


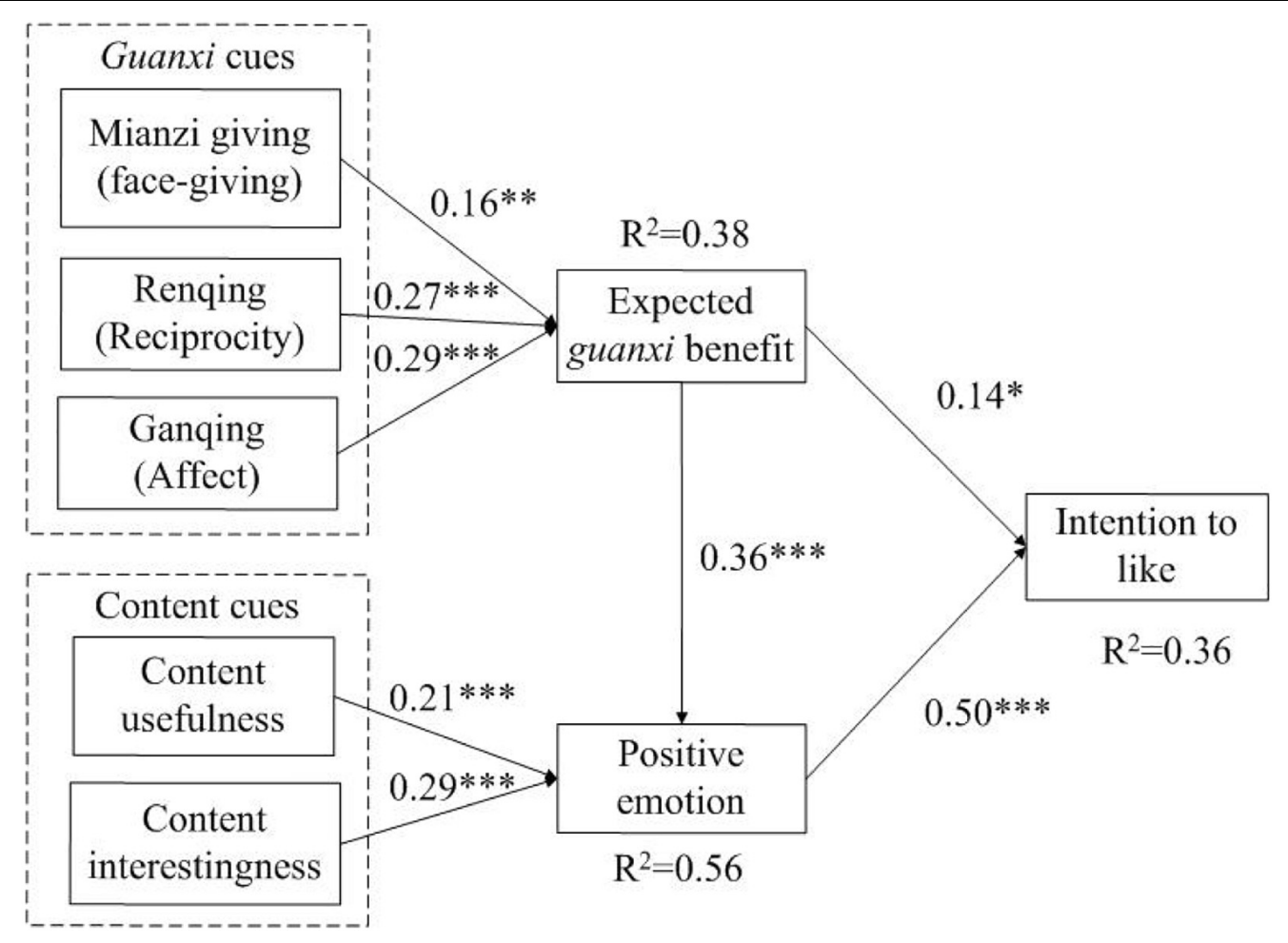

FIGURE 2 | Model testing results by SmartPLS. ${ }^{*} p<0.05,{ }^{* *} p<0.01,{ }^{* \star *} p<0.001$; n.s, not significant.

like each other's post on WeChat so as to create or maintain a desirable social image (mianzi face giving) and express their affect for close friends (ganqing). Thus, this study contributes to the field of liking behavior on social media by introducing the concept of guanxi and demonstrated the impact of social image concern and affective concerns on liking behavior.

\section{Practical Implications}

From a practical standpoint, the findings of this study are also important for social media developers to encourage Chinese users to adopt liking behavior. First, Chinese users' liking behavior can be motivated by positive emotions; that is, Chinese people are more likely to click the like button when they experience enjoyment and are in a positive emotional state. Thus, social media developers can improve Chinese users' liking behavior by designing various features to cultivate their positive emotions, such as designing the content recommendation function to provide useful and interesting content ranking lists to users, which will arouse their positive emotions. Second, Chinese users' liking behavior is also driven by motivations to maintain and enhance guanxi, with mianzi giving, renqing, and ganqing improving the expected guanxi benefit. Thus, to improve users' mianzi giving motivation, social media developers can design features to induce users' mianzi consciousness on social media (e.g., informing their friends when their posts are liked by others) and provide like ranking lists to all users. In addition, social media developers can cultivate renqing and ganqing by offering more channels to improve interpersonal interaction.

\section{Limitation and Future Research Direction}

This study also has two limitations. First, we conducted the study in China; however, the motivations underlying people's use of the like button on social media may differ across cultures. Although guanxi is a uniquely Chinese cultural construct, we assume that its underlying components such as reciprocity (renqing), affect (ganqing), social image support (mianzi face giving) should also exist and work in other culture. It would be interesting to see future research examining these underlying components and their impacts on social media liking behavior in Western culture. Second, although users' behavior is influenced by guanxi and content, liking behavior is also potentially influenced by people's personalities. Future research should consider how personal characteristics influence liking behavior.

\section{DATA AVAILABILITY STATEMENT}

The datasets generated for this study are available on request to the corresponding author.

\section{ETHICS STATEMENT}

Ethical review and approval was not required for the study on human participants in accordance with the local legislation and institutional requirements. Written informed consent for participation was not required for this study in accordance with the national legislation and the institutional requirements. 


\section{AUTHOR CONTRIBUTIONS}

$\mathrm{HZ}$ and $\mathrm{MZ}$ conceived of the presented idea, developed the theory and research model, collected data, and performed the analysis. Both authors contributed to the article and approved the submitted version.

\section{FUNDING}

The authors gratefully acknowledge financial support from the National Natural Science Foundation of China (Grant No: 71902104), Humanities and Social

\section{REFERENCES}

Ajzen, I., and Fishbein, M. (1980). Understanding Attitudes and Predicting Social Behavior. Upper Saddle River, NJ: PRENTICE-HALL.

Alston, J. P. (1989). Wa, Guanxi, and Inhwa: managerial principles in Japan, China, and Korea. Bus. Horiz. 32, 26-31. doi: 10.1016/s0007-6813(89)80007-2

Basalingappa, A., Subhas, M., and Tapariya, M. R. (2016). Understanding likes on facebook: an exploratory study. Online J. Commun. Media Technol. 6, 234-249.

Beatty, S. E., and Ferrell, M. E. (1998). Impulse buying: modeling its precursors. J. Retail. 74, 169-191. doi: 10.1016/s0022-4359(99)80092-x

Bock, G. W., Zmud, R. W., Kim, Y. G., and Lee, J. N. (2005). Behavioral Intention Formation in knowledge sharing: examining the roles of extrinsic motivators, social-psychological forces, and organizational climate. Mis Quarterly 29, 87111.

Bunker, M. P. (2013). The antecedents of satisfaction for facebook" Likers" and their effect on word-of-mouth. Proc. Market. Manage. Assoc. 23, 21-34.

Burke, K. (1966). Language as Symbolic Action. Los Angeles, CA: University of California Press.

Cardon, P. W., and Scott, J. C. (2003). Chinese business face: communication behaviors and teaching approaches. Bus. Commun. Quarterly 66, 9-22. doi: $10.1177 / 108056990306600402$

Che, H. L., and Cao, Y. (2014). Examining WeChat users' motivations, trust, attitudes, and positive word-of-mouth: evidence from China. Comp. Hum. Behav. 41, 104-111. doi: 10.1016/j.chb.2014.08.013

Chen, C. C., Chen, Y. R., and Xin, K. (2004). Guanxi practices and trust in management: a procedural justice perspective. Organ. Sci. 15, 200-209. doi: 10.1287/orsc. 1030.0047

Chen, Y., Friedman, R., Yu, E., and Sun, F. (2012). Examining the positive and negative effects of Guanxi practices. SSRN Electronic J. 28, 715-735. doi: 10. 1007/s10490-009-9176-x

Gan, C. (2017). Understanding WeChat users' liking behavior: an empirical study in China. Comp. Hum. Behav. 68, 30-39. doi: 10.1016/j.chb.2016.11.002

Gan, C., and Li, H. (2018). Understanding the effects of gratifications on the continuance intention to use WeChat in China: a perspective on uses and gratifications. Comp. Hum. Behav. 78, 306-315. doi: 10.1016/j.chb.2017.10.003

Gobel, S. M., Shaki, S., and Fischer, M. H. (2011). The cultural number line: a review of cultural and linguistic influences on the development of number processing. J. Cross Cult. Psychol. 42, 543-565. doi: 10.1177/0022022111406251

Graa, A., and Danielkebir, M. (2012). Application of stimulus \& response model to impulse buying behavior of algerian consumers. Serbian J. Manage. 7, 53-64.

Haugh, M., and Hinze, C. (2003). A metalinguistic approach to deconstructing the concepts of 'face' and 'politeness' in Chinese, English and Japanese it. J. Prag. 35, 1581-1611. doi: 10.1016/s0378-2166(03)00049-3

Hsu, C. L., and Lin, C. C. (2008). Acceptance of blog usage: the roles of technology acceptance, social influence and knowledge sharing motivation. Inform. Manage. 45, 65-74. doi: 10.1016/j.im.2007.11.001

Hwang, K. K. (1987). Face and favor: the Chinese power game. Am. J. Sociol. 92, 944-974. doi: $10.1086 / 228588$

Hwang, K. K. (1997). Guanxi and Mientze: conflict resolution in Chinese society. Int. Commun. Stud. 7, 17-38.
Sciences Foundation of the Ministry of Education, China (Grant No: 17YJC630223), China Postdoctoral Science Foundation (Grant No: 2017M612299), Shanghai Pujiang Program (Grant No: 2019PJC036), and Fundamental Research Funds for the Central Universities (Grant No: 2019ECNU-HWFW027).

\section{SUPPLEMENTARY MATERIAL}

The Supplementary Material for this article can be found online at: https://www.frontiersin.org/articles/10.3389/fpsyg. 2020.01736/full\#supplementary-material

Jacobs, J. B. (1979). A preliminary model of particularistic ties in Chinese political alliances: Kan-Ch'ing and Kuan-Hsi in a rural Taiwanese township. China Quarterly 78, 237-273. doi: 10.1017/s0305741000040467

King, Y. C. (1991). Kuan-hsi and network building: a sociological interpretation. Daedalus 120, 63-84.

Lee, D. J., Pae, J. H., and Wong, Y. H. (2001). A model of close business relationships in China (guanxi). Eur. J. Market. 35, 51-69. doi: 10.1108/ 03090560110363346

Lee, D. Y., and Dawes, P. L. (2005). Guanxi, trust, and long-term orientation in Chinese business markets. J. Int. Market. 13, 28-56. doi: 10.1509/jimk.13.2.28. 64860

Lee, L. W. Y., Tang, Y., Yip, L. S. C., and Sharma, P. (2017). Managing customer relationships in the emerging markets - guanxi as a driver of Chinese customer loyalty. J. Bus. Res. 86, 356-365. doi: 10.1016/j.jbusres.2017.07.017

Lee, S. Y., Hansen, S. S., and Jin, K. L. (2016). What makes us click "like" on Facebook? Examining psychological, technological, and motivational factors on virtual endorsement. Comp. Commun. 73, 332-341. doi: 10.1016/j.comcom. 2015.08.002

Leiner, H. C., Leiner, A. L., and Dow, R. S. (1991). The human cerebro-cerebellar system: its computing, cognitive, and language skills. Behav. Brain Res. 44, 113-128. doi: 10.1016/s0166-4328(05)80016-6

Lovett, S., Simmons, L. C., and Kali, R. (1999). Guanxi Versus the market: ethics and efficiency. J. Int. Bus. Stud. 30, 231-247. doi: 10.1057/palgrave.jibs.84 90068

Luo, Y., Huang, Y., and Wang, S. L. (2012). Guanxi and organizational performance: a meta-analysis. Manage. Organ. Rev. 8, 139-172. doi: 10.1111/j. 1740-8784.2011.00273.x

Mariani, R., and Mohammed, D. (2014). "Like" a global endorsement. how clicking "Like" influences Facebook users brand recall and future purchasing intentions. J. Manag. Policy Pract. 15, 51-63.

Mascolo, M. F., and Fischer, K. W. (2015). Dynamic Development of Thinking, Feeling, and Acting. Handbook of Child Psychology and Developmental Science. Hoboken, NJ: John Wiley \& Sons, Inc.

Mehrabian, A., and Russell, J. A. (1974). An Approach to Environmental Psychology. Cambridge, MA: MIT.

Mei-hui Yang, M. (1994). Gifts, Favors, and Banquets : the Art of Social Relationships in China. Ithaca, NY: Cornell University Press.

Nie, R., Zhong, W., Zhou, M., Jiang, W., and Wang, X. (2011). A bittersweet phenomenon: the internal structure, functional mechanism, and effect of guanxi on firm performance. Industrial Mark. Manage. 40, 540-549. doi: 10. 1016/j.indmarman.2010.12.010

Olney, T. J., Holbrook, M. B., and Batra, R. (1991). 'Consumer responses to advertising: the effects of Ad content, emotions, and attitude toward the Ad on viewing time'. J. Consum. Res. 17, 440-453.

Park, S. H., and Luo, Y. (2010). Guanxi and organizational dynamics: organizational networking in Chinese firms. Strat. Manage. J. 22, 455-477. doi: $10.1002 /$ smj. 167

Pennebaker, J. W., and Francis, M. E. (1996). Cognitive, emotional, and language processes in disclosure. Cogn. Emot. 10, 601-626. doi: 10.1080/ 026999396380079 
Redding, S. G., and Michael, N. (1983). The role of "Face" in the organizational perceptions of Chinese managers. Int. Stud. Manage. Organ. 13, 92-123. doi: 10.1080/00208825.1983.11656369

Semin, G. R., and Fiedler, K. (1988). The cognitive functions of linguistic categories in describing persons: social cognition and language. J. Pers. Soc. Psychol. 54, 558-568. doi: 10.1037/0022-3514.54.4.558

Soldat, A. S., Sinclair, R. C., and Mark, M. M. (1997). Color as an environmental processing cue: external affective cues can directly affect processing strategy without affecting mood. Soc. Cogn. 15, 55-71. doi: 10.1521/soco.1997.15. 1.55

Sun, Y., Liu, D., Shen, X. L., Zhang, X., and Wang, N. (2016). "Understanding the factors affecting users' like intentions in social network services: a multidimensional value perspective," in Proceedings of the 49th Hawaii International Conference on System Sciences (HICSS), Koloa, HI, 2286-2295.

Sussman, S. W., and Siegal, W. S. (2003). Informational influence in organizations: an integrated approach to knowledge adoption. Inform. Syst. Res. 14, 47-65. doi: 10.1287 /isre.14.1.47.14767

Tajima, Y., and Duffield, N. (2012). Linguistic versus cultural relativity: on Japanese-Chinese differences in picture description and recall. Cogn. Linguis. 23, 675-709.

Tsui, A. S., and Farh, J. L. L. (1997). Where Guanxi matters. Work Occup. 24, 56-79. doi: $10.1177 / 0730888497024001005$

Valdez, P., and Mehrabian, A. (1994). Effects of color on emotions. J. Exp. Psychol. Gen. 123, 394-409. doi: 10.1037/0096-3445.123.4.394

Verhagen, T., and Dolen, W. V. (2011). The influence of online store beliefs on consumer online impulse buying: a model and empirical application. Inform. Manage. 48, 320-327. doi: 10.1016/j.im.2011.08.001

Verhoef, P. C., Franses, P. H., and Hoekstra, J. C. (2002). The effect of relational constructs on customer referrals and number of services purchased from a multiservice provider: does age of relationship matter? J. Acad. Market. Sci. 30:202. doi: 10.1177/0092070302303002

Wang, C., Zhou, Z., Jin, X. L., Fang, Y., and Lee, K. O. (2017). The influence of affective cues on positive emotion in predicting instant information sharing on microblogs: gender as a moderator. Inform. Process. Manage. 53, 721-734. doi: 10.1016/j.ipm.2017.02.003

Wang, C. L. (2007). Guanxi vs. relationship marketing: exploring underlying differences. Indust. Market. Manage. 36, 81-86. doi: 10.1016/j.indmarman. 2005.08.002

Wang, Y., Li, Y., Semaan, B., and Tang, J. (2016). "Space collapse: reinforcing, reconfiguring and enhancing Chinese social practices through WeChat," in
Proceedings of the Tenth International AAAI Conference on Web and Social Media, Maternushaus.

Warren, D. E., Dunfee, T. W., and Li, N. (2004). Social exchange in China: the double-edged sword of Guanxi. J. Bus. Ethics 55, 355-372.

Yen, D. A., Abosag, I., Huang, Y. A., and Nguyen, B. (2017). Guanxi GRX (ganqing, renqing, xinren) and conflict management in Sino-US business relationships. Indust. Market. Manage. 66, 103-114. doi: 10.1016/j.indmarman.2017. 07.011

Yen, D. A., Barnes, B. R., and Wang, C. L. (2011). The measurement of guanxi: introducing the GRX scale. Indust. Market. Manage. 40, 97-108. doi: 10.1016/ j.indmarman.2010.09.014

Ying, F. (2002). Guanxi's consequences: personal gains at social cost. J. Bus. Ethics $38,371-380$.

Zhang, C. B., Li, Y. N., Wu, B., and Li, D. J. (2016). How WeChat can retain users: roles of network externalities, social interaction ties, and perceived values in building continuance intention. Comp. Hum. Behav. 69, 284-293. doi: 10.1016/ j.chb.2016.11.069

Zhang, P. (2013). The affective response model: a theoretical framework of affective concepts and their relationships in the ICT context. Mis Quarterly 37, 247-274. doi: $10.25300 / \mathrm{misq} / 2013 / 37.1 .11$

Zhao, H., Su, C., and Hua, Z. (2016a). Investigating continuance intention to follow a brand micro-blog: perceived value and social identification. Inform. Dev. 32, 1428-1441. doi: 10.1177/0266666915602522

Zhao, H., Su, C., and Hua, Z. (2016b). To participate or not to participate in a brand micro-blog: facilitators and inhibitors. Inform. Dev. 32, 1774-1785. doi: 10.1177/0266666915626205

Zhuang, G. J., and You-Min, X. I. (2007). Power, conflict and cooperation:the impact of personal Guanxi in a Chinese marketing channel. J. Manage. Sci. 39, 137-149. doi: 10.1016/j.indmarman.2008.07.002

Conflict of Interest: The authors declare that the research was conducted in the absence of any commercial or financial relationships that could be construed as a potential conflict of interest.

Copyright (c) 2020 Zhao and Zhang. This is an open-access article distributed under the terms of the Creative Commons Attribution License (CC BY). The use, distribution or reproduction in other forums is permitted, provided the original author(s) and the copyright owner(s) are credited and that the original publication in this journal is cited, in accordance with accepted academic practice. No use, distribution or reproduction is permitted which does not comply with these terms. 